\title{
Mycobacterium tuberculosis: Espoligotipos en el Estado Carabobo, Venezuela
}

\author{
Mónica Sequera C., Víctor Delgado S., Wolfan Araque M., Mariela Torrealba O., Raimunda Núñez M., \\ Omaira Da Mata J., Edgar Abadia P., Howard Takiff y Jacobus De Waard
}

Valencia, Venezuela: Laboratorio de Diagnóstico de Enfermedades Infecciosas (MSC, VDS,WAM)

Universidad de Carabobo insaludValencia-Carabobo (MTO, RNM) Instituto Nacional de Higiene Rafael Rangel-Caracas (EAP, HT) Laboratorio Genética Molecular IVIC Altos de Pipe Eduardo Miranda (JDW) Instituto de Biomedicina, Caracas Venezuela

Recibido: 24 abril 2007 Aceptado: 5 junio 2008

Correspondencia a: Víctor Delgado S delgadov@uc.edu.ve

\section{Mycobacterium tuberculosis: Spoligotypes in the Carabobo State, Venezuela}

In order to study the genetic variability of Mycobacterium tuberculosis circulating in the Carabobo State at Venezuela, 317 strains of M. tuberculosis isolated from patients living in different health districts whose acid fast smears were positive, were included. Each strain was characterized by spoligotyping and the pattern compared with the national and worldwide SpolDB4.0 databases; 220 isolates $(69.4 \%)$ were grouped in 24 clusters, being LAM the most common family (34\%). In contrast to other Venezuelan regions, the most common Carabobo spoligotype was number 605, with 46 isolates (14.5\%). In addition, there were $97(30.6 \%)$ orphan spoligotypes, 19 of which are found in SpolDB4.0, and 78 non described. Co-infection with human immunodeficiency virus was detected in 11 patients $(3.5 \%)$. These results show high genotypic variability of M. tuberculosis in the region, contributing with new information for a better understanding of tuberculosis transmission in Venezuela.

Key words: Tuberculosis, epidemiology, spoligotyping, PCR, Venezuela, strain 605.

Palabras clave: Tuberculosis, epidemiología, spoligotyping, RPC, Venezuela, cepa 605.

\section{Introducción}

$\mathrm{L}$ a tuberculosis (TBC) es una enfermedad infectocontagiosa re-emergente, caracterizada por elevada morbi-mortalidad en países en vías de desarrollo y en zonas urbanas de países desarrolla$\operatorname{dos}^{1}$. Un tercio de la población mundial (1,7 billones) está infectada por Mycobacterium tuberculosis, la mortalidad anual está alrededor de 2 millones de personas y la notificación es 3,3 millones de casos en el mun$\mathrm{do}^{2,3}$. En Latinoamérica la incidencia anual promedio es de 46 por 100.000, en Venezuela de 25 por 100.000, ocupando el primer lugar como causa de muerte por un único agente infeccioso ${ }^{4}$. En el Estado Carabobo la morbilidad reportada (2006) fue de 12,47 por 100.000 , con un total de 388 casos $^{5}$.

El spoligotyping (spacer oligonucleotide typing), es una técnica basada en la reacción de polimerasa en cadena (RPC) que es muy útil para discriminar cepas de M. tuberculosis, mediante la identificación de los espaciadores presentes entre las repeticiones directas en la región DR del cromosoma ${ }^{6}$. Los espaciadores son amplificados juntos e hibridizados contra 43 oligonucleótidos específicos fijados a un soporte de membrana. Los patrones revelan los espaciadores presentes en cada cepa. Esto permite discriminar entre cepas diferentes e identificar cepas iguales; de esta manera se categorizan las cepas en clusters (grupo de dos o más aislados con el mismo patrón) y cepas únicas que no tienen un patrón compartido (existente y no descritos); además, se analizan contrastando con la base de datos disponible para verificar sí el patrón ha sido reportado anteriormente en Venezuela y/o en otros países. De esta manera, los espoligotipos sirven como herramientas de apoyo en estudios sobre la dinámica de transmisión poblacional en correlación con variables clínico-epidemiológicas?

El abordaje molecular ha demostrado ser una herramienta útil en el estudio de la epidemiología mundial de la TBC. Este trabajo es el primer reporte sobre la distribución genotípica de aislados de M. tuberculosis en el Estado Carabobo en pacientes con baciloscopia positiva $\left(\mathrm{Bk}^{+}\right)$. Se describen los diferentes tipos y frecuencias encontrados y se destaca un genotipo poco común en el resto de Venezuela y raro fuera del país.

\section{Material y Métodos}

El estudio fue realizado en el Laboratorio de Diagnóstico de Enfermedades Infecciosas (LabDEI) de la Universidad de Carabobo-Valencia y el Laboratorio de 
Tuberculosis del Instituto de Biomedicina Universidad Central de Venezuela-Caracas.

Pacientes. Se obtuvieron 331 muestras de esputo de pacientes procedentes de los siete distritos sanitarios y del Centro de Referencia de TBC en el Hospital Rafael González Plaza de Valencia-Estado Carabobo. El diagnóstico directo se realizó en extendidos de esputo teñidos con técnica de Ziehl-Neelsen. Se incluyeron los casos con $\mathrm{Bk}^{+}$que aceptaron la participación en el estudio. El esputo fue descontaminado por la técnica Petroff $^{8}$ y crecido en el medio Lowenstein-Jensen. Los datos demográficos y clínicos se tomaron a partir de la ficha de notificación epidemiológica, en el período marzo 2004-diciembre 2005.

Extracción de ADN y detección. Se obtuvo 95,8\% de crecimiento en cultivo (317/331 muestras). Para la extracción de ADN bacteriano se tomaron de 4 a 5 colonias del cultivo, suspendidas en $250 \mu \mathrm{l}$ de tampón Tris-EDTA pH 8, calentado a $100{ }^{\circ} \mathrm{C}$ durante 10 min y centrifugado a $13.000 \mathrm{~g}$ por dos $\min ^{9}$, conservándose a $-70{ }^{\circ} \mathrm{C}$ hasta su uso. La detección se realizó mediante RPC. Se preparó una mezcla para 46 reacciones, de agua destilada $25 \mu \mathrm{l}$, dNTPs $(2,5 \mathrm{mM}) 2 \mu \mathrm{l}, \mathrm{MgCl}_{2}(25$ mM) $5 \mu 1$, tampón (10X) $5 \mu 1$, DRa (20 pmol) $4 \mu 1$, DRb (20 pmol) $4 \mu \mathrm{l}$ y Taq polimerasa $0,25 \mu \mathrm{l}$. La amplificación se hizo con $45 \mu \mathrm{l}$ de la mezcla y $5 \mu \mathrm{l}$ de ADN en un termociclador. Las condiciones fueron: desnaturalización por tres min a $96^{\circ} \mathrm{C}, 35$ ciclos de 1 min a $96^{\circ} \mathrm{C}$, un min a $55^{\circ} \mathrm{C}, 30$ segs a $72^{\circ} \mathrm{C}$ y al final, cinco min a $72^{\circ} \mathrm{C}$. Los cebadores usados para ampliar las secuencias espaciadoras DR fueron, DRa (GGTTTTGGGTCT GACGAC-5' biotinilado) y DRb (CCGAGAGGGGACGG $\mathrm{AAAC})^{7}$. Los productos de amplificación fueron identificados con partidores específicos para la región DR, 43 oligonucleótidos (Sigma) que se encuentran fijados a una membrana (Biodyne tipo C) y los híbridos fueron revelados por quimioluminiscencia utilizando kit ECL (enhanced chemiluminescence detection kit; Amersham, Little Chalfont, England) y autorradiografía (Hyperfilm ECL; Amersham) $)^{6,79}$. Se utilizaron como controles positivos las cepas Mycobacterium tuberculosis H37rv y Mycobacterium bovis-BCG, aisladas en el laboratorio por uno de los autores (Da Mata, O) y un control negativo (agua destilada autoclavada), que fueron tratados con el mismo procedimiento de las muestras. Los patrones se compararon con SpolDB4.0 para identificarlos, según el número de cepa atribuido a cada tipo, patrón, clase (familia) y procedencia geográfica $^{10,11}$.

Análisis estadístico. Se utilizó el software Graphpad ${ }^{12}$, aplicando la prueba de Chi cuadrado $\left(\chi^{2}\right)$ con $95 \%$ de intervalo de confianza $\mathrm{y} \pm=0,05$, para comparación de variables considerando diferencia significativa $\mathrm{p}<0,05$.

\section{Resultados y Discusión}

Los perfiles de espoligotipos de las 317 cepas, mostraron gran variabilidad, con un total de 121 patrones, agrupándose 220 aislados en 24 cluster $(69,4 \%)$, dejando 97 (30,6\%) aislados en patrones únicos, de los cuales 19 han sido reportados en la base de datos SPOLDB4. $0^{10}$ y 78 no descritos. La mayoría de los espoligotipos pertenecieron a la familia LAM (34\%).

La Tabla 1 relaciona las características clínico-epidemiológicas de los 317 pacientes en el estudio. La edad promedio fue de 38,1 años. Dos tercios $(66 \%)$ de los pacientes pertenecieron al sexo masculino, como fuera reportado en otros estudios ${ }^{13,14}$. Solamente $3 \%$ tenían co-infección VIH/TBC; aunque bajo, no deja de ser

Tabla 1. Características clínico-epidemiológicas de 317 aislados, comparando pacientes cuyas cepas tenían espoligotipos en clusters con pacientes con espoligotipos únicos

\begin{tabular}{|c|c|c|c|c|}
\hline \multirow{3}{*}{ Total pacientes } & \multicolumn{4}{|c|}{ Número $(\%)$ de pacientes con espoligotipos } \\
\hline & $\begin{array}{c}n \\
\text { (\% del total) }\end{array}$ & $\begin{array}{c}\text { En clusters } \\
(\%)\end{array}$ & \multicolumn{2}{|c|}{$\begin{array}{c}\text { Únicos } \\
\text { (\%) }\end{array}$} \\
\hline & $317(100 \%)$ & $220(69 \%)$ & & (31\%) \\
\hline \multicolumn{5}{|l|}{ Edad en años } \\
\hline Promedio (rango) & $38,15(1-92)$ & $37,5(15-92)$ & 39,6 & $(1-92)$ \\
\hline \multicolumn{5}{|l|}{ Característica } \\
\hline \multicolumn{5}{|l|}{ Sexo } \\
\hline Masculino & $210(66 \%)$ & 152 (72\%) & & $3(28 \%)$ \\
\hline Femenino & $107(34 \%)$ & 68 (64\%) & 39 & $(36 \%)$ \\
\hline \multicolumn{5}{|l|}{ VIH } \\
\hline Positivo & $11 \quad(3 \%)$ & $7(64 \%)$ & & $(36 \%)$ \\
\hline Negativo & $306(97 \%)$ & $213(70 \%)$ & 93 & $(30 \%)$ \\
\hline \multicolumn{5}{|l|}{ Baciloscopia* } \\
\hline$+1++$ & $145(46 \%)$ & $100(69 \%)$ & 45 & $5(31 \%)$ \\
\hline+++ & $172(54 \%)$ & $120(70 \%)$ & 52 & $(30 \%)$ \\
\hline \multicolumn{5}{|l|}{ Radiología } \\
\hline Normal & $13(4 \%)$ & $10(77 \%)$ & & $3(23 \%)$ \\
\hline \multicolumn{5}{|l|}{$\mathrm{LC}^{* *}$} \\
\hline Bilateral & 202 (64\%) & $143(71 \%)$ & 59 & $(29 \%)$ \\
\hline Unilateral & $102(32 \%)$ & $67(66 \%)$ & 35 & $5(34 \%)$ \\
\hline \multicolumn{5}{|l|}{ Tratamiento previo } \\
\hline Sí & 42 (13\%) & $34(81 \%)$ & & $(19 \%)$ \\
\hline No & $275(87 \%)$ & $186(68 \%)$ & & $(32 \%)$ \\
\hline \multicolumn{5}{|c|}{ Resistencia a anti-tuberculosos } \\
\hline Sí & $10(3 \%)$ & $5 \quad(50 \%)$ & & $5(50 \%)$ \\
\hline No & 307 (97\%) & $215(70 \%)$ & & (30\%) \\
\hline
\end{tabular}


importante al respecto del repunte de TBC en el mundo y en Venezuela, donde se ha reportado 0,5/100.000 de asociación TBC-VIH ${ }^{5}$. Las muestras de esputo fueron cultivada solamente si el examen microscópico resultaba positivo, y casi la mitad fueron levemente o moderadamente positivo $(\mathrm{Bk}+/++46 \%)$, mientras que la otra mitad fue muy bacilifera $(\mathrm{Bk}+++54 \%)$. Por radiología, casi dos tercios (64\%) tenían evidencia de lesiones cavernosas bilaterales, mientras que un tercio $(32 \%)$ presentaron lesiones unilaterales, y unos pocos $(4 \%)$ tenían placas de tórax normales. Trece por ciento habían recibido tratamiento para $\mathrm{TBC}$ previamente, y apenas 3\% tuvieron resistencia a anti-tuberculosos. En el análisis estadístico de comparación de pacientes con aislados en clusters y espoligotipos únicos, no se observó diferencia estadísticamente significativa (datos no mostrados); sin embargo, había unas tendencias interesantes de mencionar.

Al comparar las características de los 220 pacientes cuyos aislados tuvieron patrones de espoligotipos agrupados, con los 97 pacientes cuyos aislados tenían patrones únicos, se observó: el promedio de la edad de los pacientes con patrones únicos fue casi dos años más que los pacientes con patrones en clusters $(39,6$ versus 37,5 ), posiblemente reflejando más reactivación en pacientes mayores con cepas que no están siendo transmitidas actualmente. También hay mayor proporción de los hombres en clusters que mujeres (72\% masculino versus $64 \%$ femenino) quizás reflejando más transmisión activa entre los hombres (diferencia no significativa). No existió gran diferencia con respecto a la co-infección con $\mathrm{VIH}(70 \%$ con infección por VIH versus $64 \%$ sin infección por VIH en clusters), o bacilos vistos en muestras clínicas $(70 \%$ de los $\mathrm{Bk}+/++$ versus $69 \%$ de los $\mathrm{Bk}+++$ en clusters). Radiológicamente, tampoco se observó diferencia en los pacientes clusters (71\% cavernas bilateral, $66 \%$ unilateral y $77 \%$ normal). Las dos características con diferencias más grandes fueron en los pacientes que habían recibido tratamiento previo para $\mathrm{TBC}$, donde $81 \%$ de las cepas estuvieron en clusters, comparado con $68 \%$ de los pacientes con cepas sin tratamiento previo, y de los 10 pacientes con resistencia a, por lo menos, un fármaco anti-tuberculoso (rifampicina, isoniacida, pirazinamida y etambutol), la mitad (50\%) tenía cepas en clusters, contra $70 \%$ de los pacientes con cepas sensibles a todos los anti-tuberculosos. Esta diferencia es llamativa, pero por el pequeño número de pacientes con resistencia (10) es difícil atribuirle alguna importancia.

Los patrones más comunes, utilizando los números de identificación de shared type (ST) en el SPOLDB4.013 (Figura 1) fueron, ST605 (14,5\%), ST17 de la familia LAM 2 (12,6\%), ST93 de la familia LAM 5 (11\%) y ST42 de la familia LAM $9(8,8 \%)$. La familia LAM predomina en los países sudamericanos, como ha sido reportado por Candia y cols en Paraguay ${ }^{15}$, donde representó el $35,3 \%$ de todas las cepas, y es muy común en Europa y el Caribe. La frecuencia del tipo 605 (14,5\%) que se encontró en el Estado Carabobo es muy superior al 3$3,24 \%$ reportado en Venezuela previamente ${ }^{16,17}$. Este espoligotipo también ha sido encontrado en unos pocos aislados provenientes de otras regiones de Venezuela $^{16}$. Fuera de Venezuela, el patrón de 605 ha sido encontrado solamente en dos cepas aisladas en Nueva York ${ }^{10}$

La frecuencia del ST17-LAM 2 representa más del $50 \%$ de lo reportado en el país ${ }^{16}$, mientras que el tipo ST93-LAM 5 y ST42-LAM 9 fue superior al 12,5\% y $15,4 \%$ reportado respectivamente para sus frecuencias nacionales ${ }^{16}$. El tipo ST33-LAM 3, que se encontró en una frecuencia muy baja $(1,6 \%)$, es ligeramente superior al $1 \%$ reportado en Venezuela y ha sido descrito también en Europa y E.U.A. ${ }^{10,16}$. Los 19 patrones únicos correspondieron a los tipos: ST4, ST1209, ST866, ST1692, ST1691, ST482, ST1700, ST1685, ST81, ST37 Clase T3, ST176, ST159, ST150, ST162, ST1154, ST44, ST237, ST169 y ST395, destacándose entre ellos el tipo 482 Mycobacterium bovis- $B C G$ (caso único encontrado en una paciente de un año de edad). Es importante destacar la variabilidad genética existente entre los patrones únicos reportados y los clusters, muchos de los cuales han sido descritos por primera vez en los estudios realizados en Venezuela ${ }^{16}$, a excepción de ST1691, ST150, ST162 y ST44 que fueron descritos previamente en un estudio que abarcó todos los estados de Venezuela ${ }^{17}$. Además, se han encontrado en otros países tales como: cluster ST863 (Brasil y E.U.A.), ST1209 (México y E.U.A.), ST81 (Argentina, Brasil, Cuba e Italia), ST176 (Brasil y E.U.A.), ST159 (Argentina, Italia y E.U.A.), ST1154 (Australia y E.U.A.), ST237 (Italia y E.U.A.), ST395 (España y E.U.A.) y ST169 (Italia) $^{10}$. Los 78 patrones únicos no reportados en la base de datos internacional no reflejan significancia por cuanto fueron reportados en pacientes individuales; se debe esperar a la actualización de la base de datos y poder así confirmar si se tratan de patrones nuevos y particulares para Venezuela. También es posible que algunos de estos patrones sean el resultado de errores o artefactos; sin embargo, se intentó evitar esta posibilidad repitiendo resultados técnicamente dudosos. La frecuencia del cluster ST605 y la clase LAM difiere de otras regiones del país, sugiriendo la existencia de asociación con rutas de transmisión reciente.

En la Figura 2 se muestra la diversidad de variantes encontradas en relación con las regiones geográficas (siete distritos sanitarios). Se observó que el tipo 605 se distribuye en todo el estado, principalmente en el 


\begin{tabular}{|c|c|c|c|}
\hline$T \mathbf{m}$ & Fanc. & DESCKIPCIÓNDEESPOIGOTIPQS & $f(-6)$ \\
\hline 605 & $\mathbf{U}$ & 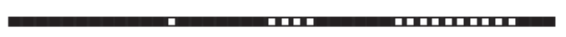 & $46(14,5)$ \\
\hline 17 & LAM 2 & $m$ & $40(12,6)$ \\
\hline 93 & LAM 5 & $\mathbf{m}$ & $35(11,0)$ \\
\hline 42 & LAM9 & $m$ & $28(8,8)$ \\
\hline 1696 & LAMS & $+\infty$ & $15(4,7)$ \\
\hline 53 & $\mathbf{T 1}$ & - & $7(2,2)$ \\
\hline 33 & LAM 3 & $\mathbf{m}$ & $5(1,6)$ \\
\hline 1702 & LAMS & man & $4(1,3)$ \\
\hline 20 & LAM 1 & $=$ & $4(1,3)$ \\
\hline 47 & Haxime & $\mathbf{m}$ & $4(1,3)$ \\
\hline NI & & & $3(0,9)$ \\
\hline 34 & $\mathbf{s}$ & 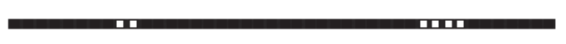 & $3(0,9)$ \\
\hline 50 & 3 & min & $3(0,9)$ \\
\hline 51 & $\mathbf{T 1}$ & m & $3(0,9)$ \\
\hline 863 & $\mathbf{U}$ & 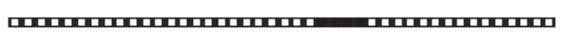 & $2(0,6)$ \\
\hline NII & & प्रा & $2(0,6)$ \\
\hline 375 & LAMS & nom & $2(0,6)$ \\
\hline NI & & שח & $2(0,6)$ \\
\hline 194 & LAM2-LAM4 & -1 & $2(0,6)$ \\
\hline 91 & $\mathbf{X 3}$ & m01000 & $2(0,6)$ \\
\hline NI & & $\mathbf{m}$ & $2(0,6)$ \\
\hline 1698 & $\mathbf{U}$ & 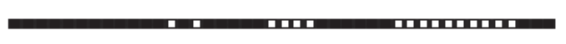 & $2(0,6)$ \\
\hline NI & & $\mathbf{m}$ & $2(0,6)$ \\
\hline 62 & Haxileml & Dmonem & $2(0,6)$ \\
\hline 4 & LAMB-S conv & 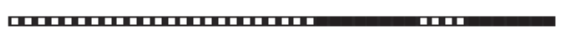 & $1(0,3)$ \\
\hline 1209 & $\mathbf{T 1}$ & 000000000000 m & $1(0,3)$ \\
\hline 1692 & $\mathbf{x 1}$ & man & $1(0,3)$ \\
\hline 866 & LAMG & m & $1(0,3)$ \\
\hline 1691 & LAM2 & 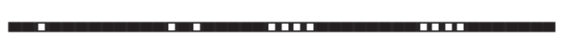 & $1(0,3)$ \\
\hline 1700 & $\mathbf{T 1}$ & m & $1(0,3)$ \\
\hline 1685 & LAMG & $m$ & $1(0,3)$ \\
\hline $\mathbf{8 1}$ & LAMG & $m$ & $1(0,3)$ \\
\hline 176 & LAMS & $\infty$ & $1(0,3)$ \\
\hline 37 & $\mathbf{T 3}$ & $\infty$ & $1(0,3)$ \\
\hline 159 & $\mathbf{T 1}$ & 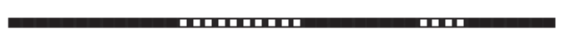 & $1(0,3)$ \\
\hline 150 & LAMG & mon & $1(0,3)$ \\
\hline 162 & LAMG & 100 & $1(0,3)$ \\
\hline 1154 & LAMG & mon & $1(0,3)$ \\
\hline 44 & TS & سm & $1(0,3)$ \\
\hline 237 & U-Haxilem3 & 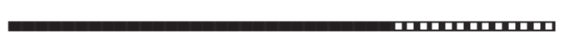 & $1(0,3)$ \\
\hline 169 & $\mathbf{U}$ & סח & $1(0,3)$ \\
\hline 395 & $\mathbf{T 1}$ & 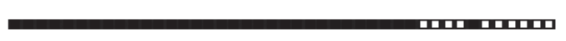 & $1(0,3)$ \\
\hline 482 & Mbovis-BCG & m & $1(0,3)$ \\
\hline
\end{tabular}

Figura 1. Representación de espoligotipos (clustery únicos reportados) en $239^{*}$ aislados de pacientes del Estado Carabobo, según tipo, familia y frecuencia porcentual. sur-oeste (18 casos) y eje de la costa (11 casos). La frecuencia en estos dos distritos sugieren las posibles vías de entrada a la cadena de transmisión, mientras que el distrito sur-oeste es el de mayor densidad poblacional (600.000 habitantes), índice de marginalidad y pobreza, y es sede de un centro penitenciario (Penal de Tocuyito). El eje de la costa (Puerto Internacional Puerto Cabello) representa una puerta de entrada a extranjeros ${ }^{18}$. El área estudiada se define como endémica, donde los casos que no se diagnostican constituyen la principal vía de propagación de la infección.

Aparte del ST 605, muchos de los otros ST comunes en el Estado Carabobo son también los más comunes en otras regiones de Venezuela. Los 17 y 93, segundo y tercero ST más común en Carabobo, son los ST más comunes en todos las otras regiones en 
Figura 2. Distribución geográfica de espoligotipos (cluster/únicos reportados) en siete distritos sanitarios del Estado Carabobo.

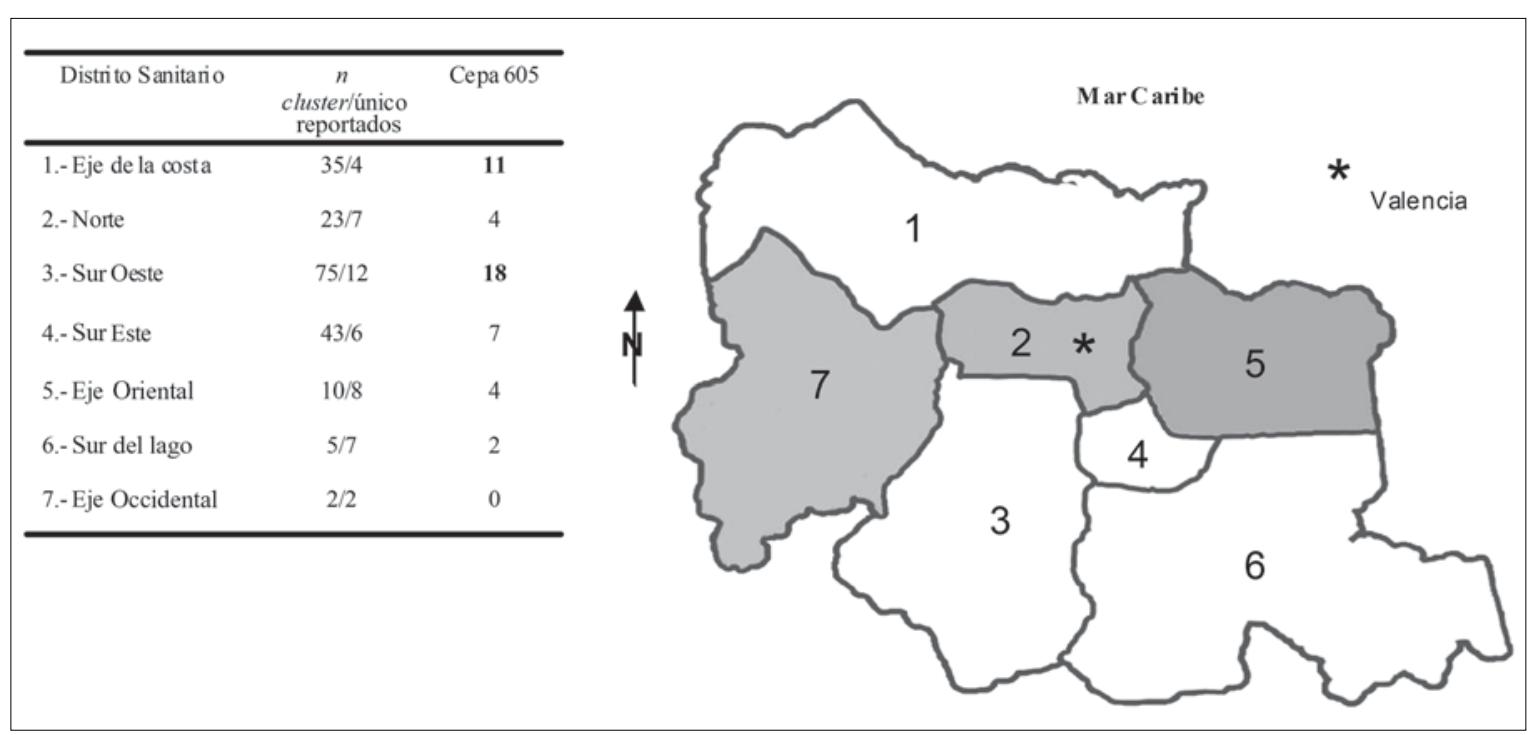

Venezuela; sin embargo, el más prevalente (ST605) no está entre los primeros siete ST más comunes en ninguna otra región. En Caracas (ubicada a unos 140 kilómetros del Estado Carabobo), se observó 20 cepas con el ST605 encontrado en más de 500 cepas analizadas $^{16}$. Mayor diferencia se obtuvo entre aislados de pacientes de la población Indígena Warao en el estado Delta Amacuro y las tribus indígenas del estado Amazonas $^{16}$.

La aplicación de técnicas de biología molecular al estudio de la transmisión de la TBC está permitiendo potenciar el programa de control para identificar puntos débiles de dicho programa. Spoligotyping es una técnica sencilla, rápida, reproducible y específica para los miembros del complejo, es un marcador genético estable y provee un patrón de diferenciación. Puede ser aplicada de manera directa en muestras clínicas e inclusive ha sido utilizado en tejido momificado, permite probar 39 cepas en un día y posee buena discriminación entre cepas con bajo número de copias IS6110. Las desventajas principales que se le atribuyen a este método es el poder discriminatorio, el cual es menor comparado con Restriction fragment length polymorphism (RFLP) IS6110 y no es completamente útil en estudios de transmisión, al menos que se combine con otra técnica9. Por esta razón, se recomienda que en investigaciones epidemiológicas se utilice una combinación de espoligotipaje y RFLP o espoligotipaje combinado con otros métodos basados en el Mycobacterial interspersed repetitive unit-variablenumber tandem repeat typing (MIRU/VNTR). La clonalidad del tipo 605 requiere del empleo de técnicas complementarias con capacidad discriminatoria como
MIRU, para aumentar la probabilidad de definir los aislados clínicos epidemiológicamente relacionados?. aislados agrupados permite una mayor diferenciación genética para precisar los genotipos causantes de la infección en la población, la cadena de transmisión, las fuentes de contaminación de la TBC y las relaciones epidemiológicas. En conclusión, se encontraron distintas variantes genéticas del bacilo. Se detectó un genotipo de M. tuberculosis con mayor frecuencia que en otros países donde la prevalencia de TBC es más alta, el ST605 que sólo ha sido reportado en E.U.A. y Venezuela. Aún cuando numerosas incógnitas requieren respuestas, la continuación del estudio epidemiológico regional requiere mayor muestreo para clarificar la importancia del abordaje molecular y la aplicación de técnicas más discriminatorias para confirmar si los casos con ST605 se trata de un brote de una sola cepa clonal.

Agradecimientos. Agradecemos la colaboración de los integrantes del Programa Regional de Control y Prevención de Tuberculosis del Estado Carabobo y a las instituciones financiadoras: Fondo Nacional de Ciencia y Tecnología (Proyectos FONACIT-2000001901 y 2005000393) y el Consejo de Desarrollo Científico y Humanístico de la Universidad de Carabobo (CDCH-99014).

\section{Resumen}

Con el objeto de estudiar la variabilidad genética de las cepas de Mycobacterium tuberculosis circulantes en el Estado Carabobo, Venezuela, se empleó la técniPosteriormente, la aplicación de RFLP y MIRU en los 
ca de spoligotyping para caracterizar 317 cepas de $M$. tuberculosis aisladas de pacientes con baciloscopia y cultivo positivo, residentes en diferentes distritos sanitarios. Los espoligotipos obtenidos se contrastaron con la base de datos nacional y mundial (SpolDB4.0); 220 aislados $(69,4 \%)$ se agruparon en 24 clusters, con predominio de la familia LAM (34\%). A diferencia de otras regiones venezolanas, el espoligotipo 605 fue el más frecuente con 46 aislados (14,5\%). Se identifica- ron $97(30,6 \%)$ aislados únicos, 19 reportados en SpolDB4.0 y 78 no descritos. Co-infección con el virus de inmunodeficiencia humana se detectó en 11 pacientes $(3,5 \%)$. Los resultados son un aporte al estudio de la transmisión de la tuberculosis a nivel regional y nacional y sugieren que existen diferencias regionales en las cepas más comunes. La cepa 605, poco común fuera de Venezuela, requiere de estudios epidemiológicos adicionales.

\section{Referencias}

1.- Kochi A. The global tuberculosis situation and the new control strategy of the World Health Organization. Tubercle 1991; 72: 16.

2.- Avilan Y, Rodríguez N, Fernández B C. Tuberculosis en el tercer milenium. Arch Hosp Vargas 2000; 44: 168-79.

3.- Raviglione M C, Zinder D E, Kochi A. Global epidemiology of tuberculosis: morbility and mortality of a worldwide epidemia. JAMA 1995; 273: 220-6.

4.- Anuarios de Estadística y Epidemiología Vital 2003; Ministerio de Salud y Desarrollo Social. Venezuela. Citado por: Hernández C, Correa MF, Zamora F et al. Aislamiento e identificación de micobacterias mediante métodos bacteriológicos y de biología molecular. Rev Soc Ven Microbiol 2005; 25 (2): 64-71

5.- MSDS. Boletín anual de situación de salud en Venezuela 2004: Disponible en: http:// www.msds.gov.ve

6.- Van Embden J D A, Cave M D, Crawford J T, Dale J W, Eisenech K D, Hermans BP, et al. Strain identification of Mycobacterium tuberculosis by DNA fingerprinting: recommendations for standardized methodology. J Clin Microbiol 1993; 31: 406-9.

7.- Kamerbeek J, Schouls L, Kolk A, van Agterveld $M$, van Soolingen D,
Kuijper S, et al. Simultaneous detection and strain differentiation of Mycobacterium tuberculosis for diagnosis and epidemiology. J Clin Microbiol 1997; 35: 907-14.

8.- Hernández C, Gómez M J. Técnicas bacteriológicas para el diagnóstico e identificación de M. tuberculosis. Acta Científica Venezolana 2001; 52: 23-5.

9. - Kremer K, van Soolingen D, Frothingham R, Haas W H, Hermans P W M, Martin C. Comparision of methods based on different molecular epidemiological markers for typing of Mycobacterium tuberculosis complex strains: interlaboratory study of discriminatory power and reproducibility. J Clin Microbiol 1999; 37: 2607-18.

10.- Brudey K, Driscoll J R, Sola C, van Soolingen D, Kremer K, Gutiérrez M C, et al. Mycobacterium tuberculosis complex genetic diversity: mining the fourth international spoligotyping database (SpolDB4) for classification, population, genetics and epidemiology. BMC Microbiology 2006; 6: 23.

11. - Filliol I, Driscoll J R, van Soolingen D, Kreiswirth B N, Kremer K, Takiff H, et al. Snapshot of moving and expanding clones of Mycobacterium tuberculosis and their global distribution assessed by spoligotyping in an international study. J Clin Microbiol 2003; 41: 1963-70.

12.- Graphpad Inc. 2005. Disponible en: http:// www.graphpad.com
13.- Urvashi B, Naga S, N. Vijaya B, Jyoti A, Hema P, Sanjeev S, et al. Predominant tuberculosis spoligotypes Delhi, India. Emerg Infect Dis 2004; 6: 1138-42.

14.- Al-Hajoj S, Zozio T, Al Rabiah F, Mohammad V, Al-Nasser M, Sola C, et al. First insight into the population structure of Mycobacterium tuberculosis in Saudi Arabia. J Clin Microbiol 2007; 45: 2467-73.

15.- Candia N, López B, Zozio T, Carrivale M, Díaz C, Russomondo G, et al. First insight into Mycobacterium tuberculosis genetic diversity in Paraguay. BMC Microbiology 2007; 7: 75.

16. - Takiff H, Abadia E, Ortega D, Sequera M, Rojas Y, Jaspe R, et al. Genotype ecology of M. tuberculosis in Venezuela. Conferencia Keystone, Tuberculosis. Whistler, Canadá, Abril 2-7, 2005.

17.- Aristimuño L, Armengol R, Cebollada A, España M, Guilarte A, Lafoz C, et al. Molecular characterization of Mycobacterium tuberculosis isolates in the First National Survey of Anti-tuberculosis Drug Resistance from Venezuela. BMC Microbiol 2006; 6: 90.

18.- Reporte de estadística y epidemiología anual del Programa Regional de Control y Prevención de Tuberculosis año 2006. Disponible en: Archivos en físico del Programa Nacional de Salud Respitaroria, Caracas-Venezuela. 